\title{
NOTA TÉCNICA DA AGÊNCIA NACIONAL DE VIGILÂNCIA SANITÁRIA No 04/2020: OPORTUNIDADES DE APERFEIÇOAMENTO E AMPLIAÇÃO DA PROTEÇÃO PARAA ENFERMAGEM
}

Marisa Aparecida Amaro Malvestio ${ }^{1}$ Eduardo Fernando de Souza ${ }^{1}$ https: //orcid.org/0000-0003-0633-9278

https: //orcid.org/0000-0002-2108-6507

Objetivos: Apresentar e analisar oportunidades de aperfeiçoamento das medidas de proteção previstas na Nota Técnica 004/2020 da Agência Nacional de Vigilância Sanitária do Ministério da Saúde do Brasil, em sua 4ạ. atualização, com vistas a promover maior proteção aos profissionais de saúde, em especial, da Enfermagem. Método: Análise argumentativa e propositiva da nota da ANVISA embasada em documentos técnicos nacionais e internacionais. Discussão: São apresentadas 10 sugestões de aperfeiçoamento que envolvem a incorporação e revisão de medidas de prevenção e controle de exposição nos diferentes ambientes de cuidado. Considerações Finais: É necessário aperfeiçoar rapidamente os documentos técnicos. Orientações assertivas e oportunas auxiliam os gestores na composição de um plano de contingência eficaz na prevenção e controle da COVID 19. Descritores: Infecções por Coronavírus; Controle de Doenças Transmissíveis; Equipamento de Proteção Individual.

TECHNICAL NOTE FROM NATIONAL HEALTH SURVEILLANCE AGENCY IN BRAZIL NO. 04/2020: OPPORTUNITIES FOR IMPROVING AND EXPANDING PROTECTION FOR NURSING

Objectives: To present and analyze opportunities for improving the protection measures provided for in the Technical Note 004/2020 of the National Health Surveillance Agency of the Ministry of Health of Brazil, in its 4th. update, with a view to promoting greater protection for nursing professionals. Method: An argumentative and purposeful analysis of the note of National Health Surveillance Agency in Brazil based on national and international technical documents. Discussion: 10 suggestions for improvement are presented that involve the incorporation and review of prevention and exposure control measures in different care environments. Final Considerations: It is necessary to improve technical documents quickly. Assertive and timely guidelines assist managers in composing an effective contingency plan in the prevention and control of COVID 19.

Descriptors: Coronavirus infections; Communicable Disease Control; Personal Protective Equipment.

NOTA TÉCNICA DA AGÊNCIA NACIONAL DE VIGILÂNCIA SANITÁRIA DO BRASIL NO. 04/2020: OPORTUNIDADES PARA MEJORAR Y AMPLIAR LA PROTECCIÓN DE ENFERMERÍA

Objetivos: Presentary analizar oportunidades para mejorar las medidas de protección previstas en la Nota técnica 004/2020 de la Agencia Nacional de Vigilancia Sanitaria del Ministerio de Salud de Brasil, en su 4to. actualización, con el fin de promover una mayor protección para los profesionales de enfermería. Método: Análisis argumentativo y resuelto de la nota de Agência Nacional de Vigilância Sanitária do Brasil basada en documentos técnicos nacionales e internacionales. Discusión: se presentan 10 sugerencias de mejora que implican la incorporación y revisión de medidas de prevención y control de exposición en diferentes entornos de atención. Consideraciones finales: es necesario mejorar rápidamente los documentos técnicos. Las pautas asertivas y oportunas ayudan a los gerentes a componer un plan de contingencia efectivo en la prevención y control de COVID 19. Descriptores: Infecciones por Coronavirus; Control de Enfermedades Transmisibles; Equipo de Protección Personal.

${ }^{1}$ Comissão Nacional de Urgência e Emergência do COFEN, Brasilia, DF.

Autor Correspondente: Marisa Aparecida Amaro Malvesio Email: marisa.malvestio@gmail.com

Recebido: 05/6/2020 Aceito: 28/5/2020 


\section{INTRODUÇÃO}

A Nota Técnica 004/2020 da Agência Nacional de Vigilância Sanitária (ANVISA) do Ministério da Saúde ${ }^{1}$, apresenta as medidas de prevenção e controle de infecção na assistência à casos suspeitos ou confirmados de infecção pelo SARS-CoV-2, a serem aplicadas em diferentes serviços de saúde no Brasil. Após 4 atualizações, desde sua publicação em 30/01/2020, a nota reflete o conhecimento incorporado ao longo do tempo até 08/05/20201. É relevante considerar que as orientações versam sobre um quadro mínimo aceitável de medidas e que os profissionais e os serviços de saúde podem implementar ações adicionais de acordo com as necessidades percebidas e os recursos disponíveis ${ }^{1}$.

O vírus SARS-Cov-2 requer simultaneamente precauções de contato, gotículas e aerossóis em 100\% do tempo e para $100 \%$ dos profissionais de saúde da linha de frente, que estão frequentemente expostos ${ }^{1-5}$. A proteção de alto nível para esses profissionais, é fundamental para preservar o indivíduo e a força de trabalho, e também para reduzir a disseminação nas instituições e na comunidade, pois não há medidas profiláticas melhores e tão pouco, medidas terapêuticas bem estabelecidas e eficazes para seu tratamento ${ }^{3.4}$. Nesse cenário de incertezas, repassar aos serviços ou aos profissionais, a decisão de incorporar medidas adicionais de proteção, se possível, não é uma ação positiva na batalha pela proteção de todos, igualmente, gerando ainda mais incertezas e grande variação.

Os profissionais de saúde precisam receber informações adequadas e atualizadas sobre o nível de proteção que necessitam e os cuidados que devem tomar. Para os serviços, a orientação técnica assertiva é importante para orientar a decisão gestora na definição de planos de ação em cada cenário.

Aos 100 dias de pandemia da COVID-19 completados em 18/06/2020, o Observatório de Enfermagem do Conselho Federal de Enfermagem já registrava cerca de 20 mil casos da doença em seus profissionais e 209 óbitos. Diante de um cenário futuro que mostra um longo período de enfrentamento e recuperação, nos cabe perguntar: Como podemos aperfeiçoar as medidas DE proteção e ir além do mínimo recomendável para proteger, mais e melhor, todos os profissionais da linha de frente?

\section{Objetivo}

Apresentar e analisar oportunidades de aperfeiçoamento das medidas de proteção previstas na Nota Técnica 04/2020 da ANVISA ${ }^{1}$, com vistas a promover maior proteção aos profissionais de Enfermagem.

\section{MÉTODO}

Análise argumentativa e propositiva da Nota Técnica 004/2020 da ANVISA ${ }^{1}$ embasada em documentos técni$\cos$ nacionais ${ }^{3}$ e internacionais, principalmente aqueles oriundos do Centro Europeu de Controle de Doenças ${ }^{3} \mathrm{e}$ da Organização Mundial de Saúde ${ }^{4}$.

\section{DISCUSSÃO}

O acompanhamento das recomendações nacionais e internacionais até a presente data, permite destacar e analisar 10 oportunidades de aperfeiçoamento na opinião dos autores:

-Incorporação de medidas de prevenção e controle de exposição no ambiente pré-hospitalar que considerem todo o contexto da atenção, desde a central de regulação, a preparação do carro e das equipes, até os cuidados na ambulância e na transição do cuidado, incluindo cuidados com a limpeza terminal da ambulância e na transferência entre unidades $^{2}$.

- Organização das recomendações sugeridas, segundo a modalidade de cada medida: Medidas ambientais e de engenharia, sanitárias, administrativas e de orientação e educação, para facilitar a composição de um plano de ação em cada serviço de saúde, respeitadas as peculiaridades ${ }^{2-4}$;

- Estabelecimento de recomendações para vigilância ativa de profissionais da saúde com acolhimento, recepção e avaliação já à entrada das unidades, seguidos de monitoramento e encaminhamento se necessário ${ }^{2-4}$;

- Definição da lista de procedimentos/situações geradores de aerossóis, acrescentando os tratamentos que envolvam nebulização, traqueostomia, desconexão do paciente do ventilador, posicionamento prona, uso de dispositivos supraglóticos, endoscopia, extubação, uso de sistema Venturi e procedimentos odontológicos dentre outros ${ }^{1,2,4}$

- Revisão da recomendação de uso de máscara de tecido por profissionais de recepção, seguranças e condutores de ambulâncias, considerando as novas recomendações de uso de máscara cirúrgica por profissionais que atuam em ambientes de saúde. É alto o potencial de exposição ao vírus nessas atividades e máscaras de tecido são itens pessoais, cuja higienização é realizada de forma doméstica e sem garantia ou controle de processo, gerando risco po- 
tencial de disseminação e exposição na unidade e na comunidade ${ }^{2-4}$;

- Revisão e uniformização das orientações sobre o conjunto de equipamentos de proteção individual (EPI) indicado para cada atividade, uma vez que a situação de escassez ou dificuldade destes EPIs já foi vencida e esses itens são essenciais para o controle e mitigação da exposição dos profissionais e da comunidade. Entidades internacionais já firmaram convicção sobre o conjunto de EPI necessários para profissionais que atuam em setores onde são realizados procedimentos geradores de aerossóis (PGA) e não apenas durante sua realização. A manutenção de dois níveis de proteção (com PGA e sem PGA), em um mesmo ambiente de risco, fragiliza o controle dos processos de cuidado, eleva o consumo de itens, resulta em guarda inadequada ou improvisada das máscaras N95 e gorros e potencialmente amplia a exposição dos profissionais. Esse conjunto mínimo deve ser composto por respirador N95 (ou equivalente), avental impermeável longo, óculos e/ou protetor facial e gorro para todos os profissionais, (incluindo, profissionais de limpeza e condutores de ambulância). A uniformização é necessária para adequar as orientações existentes orientações da própria Nota Técnica, contidas no quadro "Quem deve usar a N95" e na orientação de equipes exclusivas em áreas de Unidade de Terapia Intensiva (UTI). A orientação técnica assertiva com uma descrição adequada é importante para a decisão gestora de definição de protocolos e compras ${ }^{1-2,4}$;

- Análise e detalhamento das recomendações sobre a vestimenta tipo macacão, com vistas a realizar uma descrição orientativa para os serviços que decidirem por sua implementação. Embora o uso deste tipo de vestimenta não seja indicado por instituições internacionais, ele vem sendo utilizado por inúmeros serviços no Brasil e em todo o mundo, como forma de elevação do nível de proteção dos profissionais, inclusive em outras atividades não relacionadas ao cuidado direto. A variação de oferta e demanda associada a ausência de padrões, geram riscos e elevam a percepção de insegurança dos profissionais, principalmente para aqueles que não tiveram acesso à vestimenta, seja por uma decisão técnica, política ou por desconhecimento dos gestores ${ }^{2}$;

- Divulgação de orientações para profissionais de saúde, quanto a cuidados com higiene pessoal e limpeza/higiene de vestimentas pessoais, antes, duran- te e após os plantões (sapatos, botas, roupas, adornos e uniformes), mesmo que algumas orientações já sejam previstas em outras normas ${ }^{2.3}$;

- Complementação da descrição do avental impermeável, uma vez que existem múltiplos tipos, acrescentando características importantes para a proteção dos profissionais como: área a ser coberta, (região do colo, braços, tórax anterior e posterior, abdome, quadril e membros inferiores até os joelhos), design (gola careca, mangas longas, punho de malha ou elástico, abertura posterior, com duplo fechamento trespassado nas costas por tiras costuradas no pescoço e cintura, comprimento mínimo $130 \mathrm{~cm}$ ), propriedades (atóxico, impermeável, hipoalérgico, com baixo desprendimento de partículas e resistente), indicação de uso (proteção da pele e a roupas do profissional em situação de risco real ou potencial de exposição a fluidos corporais, incluindo gotículas e aerossóis), material (produzido em TNT, gramatura mínima $50 \mathrm{~g} / \mathrm{m} 2$ ou superior, ou material similar impermeável), e principalmente, opções de uso, em caso de escassez ou indisponibilidade ${ }^{2}$;

- Análise, atualização e complementação das medidas sugeridas para as unidades de terapia intensiva e nos serviços de emergência, a partir das recomendações desenvolvidas por sociedades de especialistas no campo ressuscitação cardiopulmonar, intubação orotraqueal, uso de cápsula acrílica e de dispositivos acessórios, dentre outros ${ }^{5}$.

\section{Limitações do estudo}

Artigos de opinião têm um caráter argumentativo e apresentam e/ou discutem a posição de seus autores sobre um tema sem uso de estratégias exaustivas de busca. Desta forma, a seleção dos aspectos analisados está sujeita a maior subjetividade è experiência dos autores.

\section{Contribuições para a prática}

O aperfeiçoamento das medidas de prevenção e controle de infecção na assistência, pode ampliar oportunamente a proteção dos profissionais de Enfermagem e demais profissionais da saúde.

\section{CONSIDERAÇÕES FINAIS}

Profissionais de saúde adoecem e morrem a cada momento de atraso em uma medida ou decisão que envolva o controle e as ações de prevenção contra a COVID-19. É necessário aperfeiçoar os documentos orientativos rapidamente, a partir de experiências nacionais e internacio- 
nais, estimulando os gestores na composição e aplicação de um plano de contingência assertivo e oportuno em cada serviço de saúde, respeitadas as peculiaridades.

Rapidez e adequação podem nascer da parceria entre órgãos reguladores, órgãos de classe e entidades de especialistas construindo um olhar particular e aprofundado sobre cada área. Orientações podem se concretizar em novos documentos eletrônicos de fácil acesso, contemplando conteúdos específicos, sempre com validação da ANVISA.

O regime de escassez de EPI não se aplica mais à con- dição atual do Brasil, uma vez que a disponibilidade desses itens vem se normalizando. No entanto, permanecemos na perspectiva de utilização da capacidade máxima das unidades de saúde em muitos estados e por mais tempo. Precisamos ir além de informações e EPI mínimos e aperfeiçoar a proteção de todos os profissionais em todos os contextos e por todo o período necessário.

Contribuições dos Autores: MAAM e EFS participaram de todas as etapas do artigo.

\section{REFERÊNCIAS}

1. Ministério da Saúde (BR). Agência Nacional de Vigilância Sanitária. Nota Técnica 004/2020 de 30 de janeiro de 2020 (atualização em 4 em 08 de maio de 2020). Orientações para serviços de saúde: medidas de prevenção e controle que devem ser adotadas durante a assistência aos casos suspeitos ou confirmados de infecção pelo novo coronavírus (Sars-cov-2) [Internet]. Brasilia (DF): Ministério da Saúde; 2020 [Citado 2020 Mai 15]. Disponivel em: http://portal.anvisa.gov.br/documents/33852/271858/Nota+T\%C3\%A9cnica+n+04-2020+GVIMS-GGTES-ANVISA/ab598660-3de4-4f14-8e6f-b934lc196b28.

2. Guimarães, HP, Damasceno MC, Ribera, JM, Onimaru, A, Malvestio, M, Bueno M, et al. Recomendações para o atendimento de pacientes suspeitos ou confirmados de infecção pelo novo coronavírus (SARS-CoV-2) pelas equipes de atendimento pré-hospitalar móvel. Associação Brasileira de Medicina de Emergência - ABRAMEDE. [Citado 2020 Mai 15] Disponivel em: http://abramede.com.br/wp-content/uploads/2020/04/RECOMENDACOES-APH-220420.pdf

3. European Centre for Disease Prevention and Control. Infection prevention and control and preparedness for COVID-19 in healthcare settings Fourth update. Stockholm: ECDC; 2020. [cited 202004 July]. Available from: https://www.ecdc.europa.eu/sites/default/files/documents/Infection-prevention-and-control-in-healthcare-settings-COVID-19_4th_update.pdf

4. World Health Organization (WHO). Infection prevention and control during health care when coronavirus (Covid 19) is suspected, Interim guidance: WHO; 2020. [cited 202030 June]. Available from: https://www.who.int/ publications/i/item/WHO-2019-nCoV-IPC-2020.4

5. Associação de Medicina Intensiva Brasileira (AMIB). Recomendações da Associação de Medicina Intensiva Brasileira para a abordagem do COVID-19 em medicina intensiva. [Citado 202030 June]. Disponivel em: https:// www.amib.org.br/fileadmin/user_upload/amib/2020/maio/28/Recomendaco__es_AMIB-2a_atual.-28.05.pdf 